\title{
A Direct Simulation Monte Carlo Approach on the Riemann Problem for Gas Mixtures
}

\author{
Stavros Meskos, a) and Stefan Stefanov ${ }^{1, b)}$ \\ ${ }^{1}$ Institute of Mechanics, Bulgarian Academy of Sciences - Acad. G. Bontchev St., bl. 4, 1113 Sofia, Bulgaria \\ a)stameskos@imbm.bas.bg \\ ${ }^{b)}$ stefanov@imbm.bas.bg
}

\begin{abstract}
The Direct Simulation Monte Carlo method is employed to solve the "1D - Sod-Shock tube problem", a special case of Riemann problems, for gas mixtures. Initially, two different gas species are distributed separately in the high and low pressure sides of the tube without interacting with each other. For time greater than zero the species start mixing and shock and rarefaction waves are formed moving in opposite directions. In this work, the mixing process between different kinds of gas species was investigated by observing differences in waves' formation. The influence of the mass and diameter ratio between the species was studied in detail. It was found that greater mass differences had very strong effect on the mixing process, while the effects due to diameter differences were relatively small. Finally, it is shown that in case of single species the interaction molecular models of hard sphere, variable hard sphere and variable soft sphere gave the same results, while for gas mixtures the variable hard sphere and variable soft sphere models gave slightly different results.
\end{abstract}

\section{INTRODUCTION}

Gas flows in micro-scale have received increasing attention in the past two decades. Advances in micro and nano-fabrication technology have opened the road for Micro-Electro-Mechanical-Systems (MEMS) and NanoElectro-Mechanical-Systems (NEMS) [1-4]. Such devices, depending on the application, can operate with certain kind of fluids. Very common working fluids are gases and so far single gas flows in micro scale have been extensively studied [5-12]. However, only recently the focus has shifted to gas mixtures and the process of gas mixing that are frequently met in real applications [13-20]. MEMS refer to devices with characteristic lengths starting as low as $1 \mu \mathrm{m}$ up to $1 \mathrm{~mm}$, therefore very often the characteristic length of the problem in study is critically small, comparable to the mean free path of the gas molecules. In such cases, the continuum assumption of the fluid is no longer valid and the classical Navier-Stokes equations cannot describe accurately the flow field. Similar conditions at higher Knudsen numbers are also met in large-scale spaces when the pressure is very low. For both cases mentioned above, where the flow depart from continuum regime, other approaches have been employed and amongst them, the most common are the deterministic Discrete Velocity (DVM) [21] and Lattice Boltzmann (LBM) [22] methods that solve the Boltzmann equation and the Direct Simulation Monte Carlo (DSMC), a stochastic particle- based method [23]. In this work, the DSMC method is employed to investigate the gas mixing process at the Sod-Shock tube problem, which is a form of the well- known Riemann problem in continuum mechanics with existing analytical solution in the case of single gas [24]. Although the Riemann problems have been extensively studied and there is a plethora of published work, there are only a few papers that investigate the same problems with numerical methods employed in slip and rarefied regimes [25-28]. To the authors best knowledge it is the first time that a Riemann problem for gas mixtures is solved with the DSMC method. 


\section{PROBLEM STATEMENT}

The Riemann problem in gas dynamics refers to a specific initial value problem defined in space $[0,1]$ with conditions given by

$$
[\rho, u, p]=\left\{\begin{array}{llll}
\rho_{L} & u_{L} & p_{L}, & \text { for } x \leq 0.5 \\
\rho_{R} & u_{R} & p_{R}, & \text { for } \mathrm{x} \geq 0.5
\end{array}\right.
$$

A mechanical realization of the Riemann problem is a shock tube. Such tube consists of a long tube with a diaphragm in the middle. Initially, the volume to the left is filled with gas having density and pressure $\rho_{L}$ and $p_{L}$ respectively, while volume to the right of the diaphragm is filled with gas having density and pressure $\rho_{R}$ and $p_{R}$ respectively. The corresponding bulk velocities at the areas right and left of the diaphragm $u_{L}$ and $u_{R}$ respectively, can be both or individually greater, less or equal to zero. The particular configuration that is investigated in this work is the Sod shock tube problem, originally studied by Gary A. Sod (1978) [24] with specific initial values defined as

$$
[\rho, u, p]=\left\{\begin{array}{cccc}
1 & 0 & 1 & \text { for } x \leq 0.5 \\
0.125 & 0 & 0.1, & \text { for } \mathrm{x} \geq 0.5
\end{array}\right.
$$

A schematic diagram of the initial condition before the diaphragm is removed is provided in Fig. 1. Figure 2 depicts a quality plot of pressure at time t equal to zero, where numbers 1 and 5 correspond to the initial high and low pressure areas respectively. At time $t=0$ the diaphragm is suddenly removed and there is an initial discontinuity in the contact surface. Immediately after that a shock and a rarefaction waves are formed, where the shock is traveling from high to low pressure area, while the rarefaction wave is traveling on the opposite direction. For $t>0$ the states of the flow can be described in five different areas as depicted in Fig. 3. Zones 1 and 5 correspond to the undisturbed high and low density states keeping their values from the initial condition. The head and foot of the rarefaction and shock wave are located at the interfaces between zones 1 and 2 and zones 2 and 3 and respectively between zones 4 and 5 and 3 and 2. Finally, the contact surface, which also travels towards the low pressure area, following the shock head lies inside the shock wave and it is located at the interface between the zones 3 and 4. Density and temperature are discontinuous within the zones surrounding the contact surface while pressure and velocity are constant. The process in zone 2 is isentropic and the flow properties gradually change.

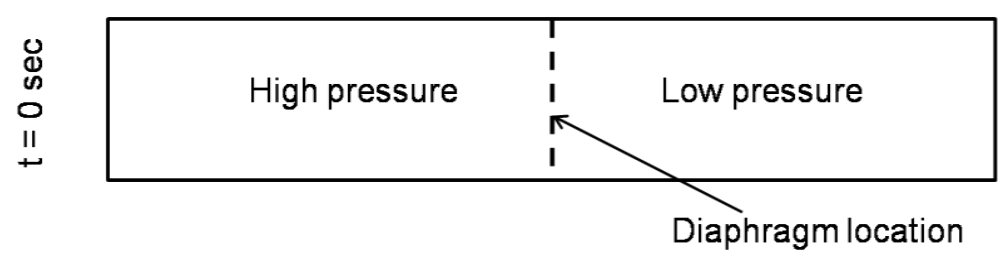

Figure 1. Schematic diagram of the initial condition of the Sod-shock tube problem.

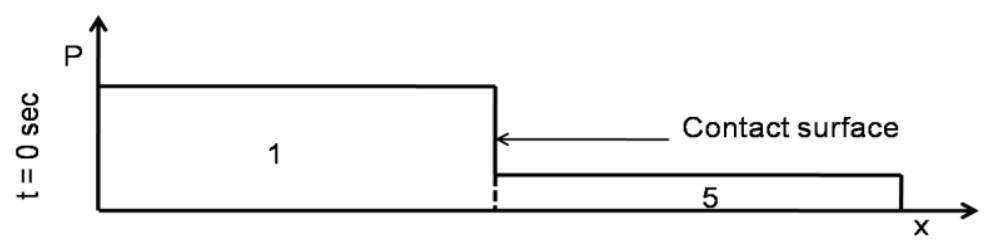

Figure 2. Quality plot of pressure before the diaphragm is removed. 


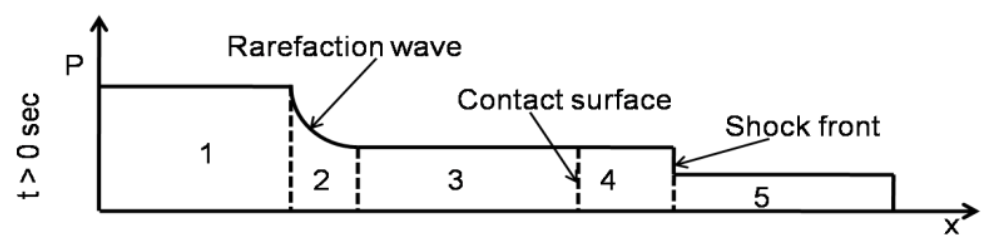

Figure 3. Quality plot of pressure after removing the diaphragm.

\section{NUMERICAL METHOD AND CODE VALIDATION}

\section{Flow regimes and mathematical formulation}

In general, the gas flow is characterized by the Knudsen number, which is defined as ratio of mean free path to characteristic length of the problem and reads as follows

$$
K n=\frac{\lambda}{L}
$$

where the mean free path $\lambda$ is given by

$$
\lambda=\frac{1}{\sqrt{2} \pi d^{2} n}
$$

$d$ is the diameter of the molecule and $n$ is the number density, related to mass density $\rho$ and molecular mass $m$ by

$$
\rho=n m .
$$

The equation of state is given by

$$
p=n k_{B} T,
$$

where $k_{B}=1.380649 \times 10^{-23}[\mathrm{~J} / \mathrm{K}]$ is the Boltzmann constant.

A continuum regime is assumed for flows with Knudsen number $K n \leq 10^{-3}$. The gas flows with Knudsen numbers in the range of $10^{-3}<K n<10^{-1}$ are considered to be in slip regime, a moderate rarefied area, where velocity slip and temperature jump at wall strongly influences heat transfer [29]. Finally, for Knudsen numbers $K n \geq 10^{-1}$ a transient rarefied flow regime is defined up to free molecular flow. In this work, it is also important for a gas mixture to define the molar fraction of species $\alpha$ by

$$
C_{a}=\frac{n_{a}}{n}
$$

where

$$
n=\sum_{\alpha=1}^{2} n_{\alpha}
$$

\section{The Direct Simulation Monte Carlo method}

The DSMC is a particle-based stochastic method that can simulate gas flows in the whole spectrum of the Knudsen regime. It is worth noting that it is extremely efficient for flows in slip and rarefied regimes but becomes 
computationally very expensive in continuum regime. The main principle of the method is the decoupling of the motion and collision processes of the particles within each time step of the numerical simulation. The computational domain is divided into cells. The simulated number of particles is a fraction of the real number; a weighted number density is defined as

$$
n_{w}=\frac{n}{F_{w}}
$$

where $F_{w}$ is the weight factor. Initially, particles are randomly distributed in cells with molecular velocities sampled from the Maxwellian distribution. Next, within a time step the particles are moved through the cells proportionally to their velocity, then collisions only between particles within the same cell are realized stochastically by using collision probability distribution and finally, macroscopic gas properties such as average flow velocity and number densities are sampled at each cell. This process is repeated each time step and the simulation continues in time until steady-state conditions are reached. There are two possibilities for averaging. The first employs time averaging of accumulated data over a time interval of finite number of time steps $k \times d t$ that corresponds to one sample step. This averaging method is usually sufficient when only the steady-state solution is of interest. However, in order to derive accurate results for the transient period as well, an ensemble averaging method is employed along with the time averaging method, where many independent simulations are performed and results are additionally averaged at the same sample steps by ensemble averaging over all simulations. The collision scheme, employed in this work is the No-Time-Counter and particles are modeled as hard spheres [23]. An in-house DSMC code was developed and employed in this work, which combines both time and ensemble averaging methods.

\section{Code Validation}

The classical 1D Sod-Shock tube problem is considered. Before proceeding to the actual gas mixing problem the correctness of the code was verified by replicating a case of a single gas as presented by Tiwari et al. [25] and comparing the results with both theirs as well as with the analytical solution. The following parameters have been used. The gas is chosen as Argon with molecular mass $m=6.63 \times 10^{-26} \mathrm{Kg}$, diameter $d=3.68 \times 10^{-10} \mathrm{~m}$, the ratio of specific heats $\gamma=5 / 3$. The temperature given initially at both sides of the tube is kept fixed approximately at $T_{L}=T_{R}=273 \mathrm{~K}$, while the mass densities are $\rho_{L}=1 \times 10^{-4} \mathrm{Kg} / \mathrm{m}^{3}$ and $\rho_{R}=0.125 \times 10^{-4} \mathrm{Kg} / \mathrm{m}^{3}$, with $\mathrm{Knudsen}$ numbers approximately equal to $K n_{L}=1 \times 10^{-3}$ and $K n_{R}=8 \times 10^{-3}$ for the left and right area respectively. The characteristic length $L=1 \mathrm{~m}$, is the total length of the tube, while the diaphragm position is at $x=0.5 \mathrm{~m}$. Total collision cells where 1200 and the sampling was performed from the averaged values of every 6 neighboring cells. The solution corresponds to time $t=6.8 \times 10^{-4} \mathrm{sec}$. This particular configuration as described above will be referred to as the "base configuration". In Fig. 4 the mass density evolution is plotted over the length of the tube, with the dashed line corresponding to the current work, while with dotted and solid lines to the Tiwari and analytical solutions respectively. The results of this works are in very good agreement with the others. It is important to notice, that the perfect sharp discontinuities as calculated by the analytical solution are impossible to retrieve with the numerical methods. Instead the interfaces between the otherwise distinctive zones are smooth transitions from one flow state to another. Finally, with the analytical solution the exact positions of those states are measured as follows, the head and foot of the rarefaction at $x_{H R}=0.29 \mathrm{~m}$ and $x_{F R}=0.46$ respectively, the contact discontinuity at $x_{C D}=0.63 \mathrm{~m}$ and the head of the shock wave at $x_{H S}=0.81 \mathrm{~m}$.

\section{From continuum to rarefied regime}

For the next test case, the base configuration was used. However the mass densities in both sides were reduced each time by a factor of ten while keeping the same ratio between them. The evolution of mass densities was compared between flows in continuum, slip and rarefied regimes. Figure 5 depicts density profiles of the three cases corresponding to Knudsen numbers in the left side at $10^{-3}, 10^{-2}$ and $10^{-1}$, with solid, dotted and dashed lines respectively. It is obvious from the plots that the classical Riemann problem is valid only in continuum regime and in the slip regime the discontinuities are vanished. 


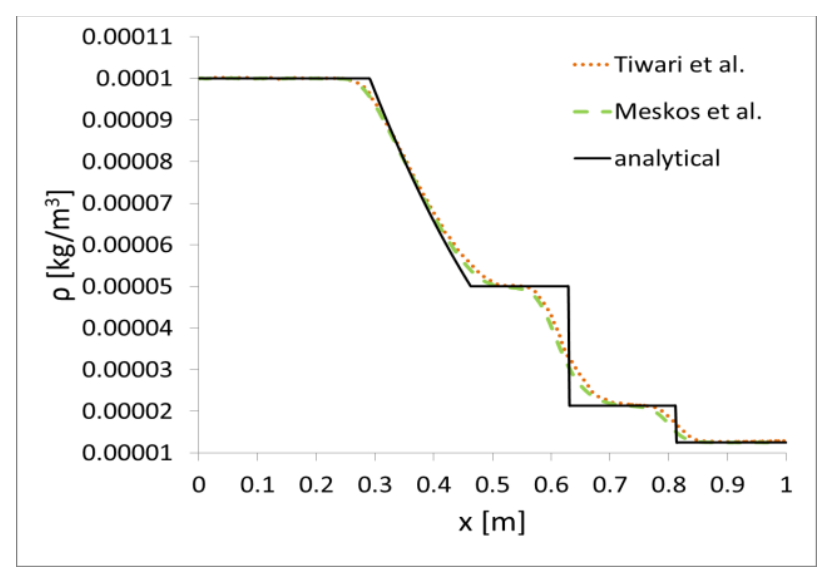

Figure 4. Mass density profiles referring to the validation case. The results of this work are depicted with the dashed line. With doted are the results of Tiwari et al. and with the solid line the analytical solution.

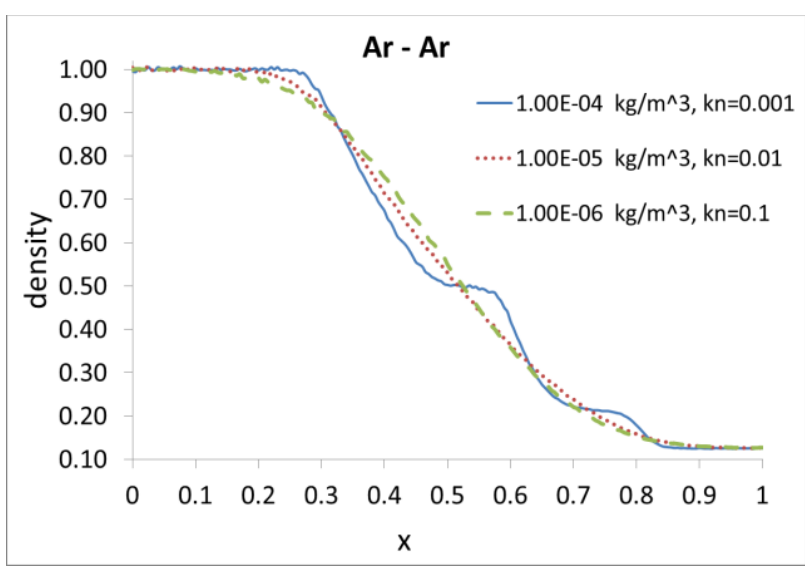

Figure 5. Dimensionless density profiles for different Knudsen numbers, covering flows from continuum up to slip and rarefied regimes.

In the following sections the results for gas mixtures are presented and organized as follows: In the first part the comparison is conducted over pairs between four noble gases. Next, follows a series of tests to investigate the mass ratio and diameter ratio influence to the mixing process independently. Finally, the impact of the three most commonly employed phenomenological molecular models, namely the hard sphere, the variable hard sphere and the variable soft sphere is investigated on a single gas, as well as, on a gas mixture case.

\section{RESULTS}

\section{Mixing of noble gases}

Following the base configuration as described in the previous section, simulations with different gas species were conducted by letting on the left side of the diaphragm, where the high density area lies, the gas Argon, as was before, while replacing it, on the right side, the low density area, with Neon, Helium, and Xenon respectively. The cases that are tabulated in Table 1 were chosen in purpose in order to cover a wide range of mass and diameter ratios. The corresponding mass density profiles are depicted in Fig. 6. It was immediately noticed that the profiles were very different from each other. With a more careful observation two differences were noticed. The value of density between the foot of the shock wave and the contact surface, marked in the previous section as zone 3, is increased when the gas in the right side is lighter and with smaller diameter e.g. Ne, He, while it is decreased for the case of the heavier Xe. The second observation refers to the contact surface and more generally to the formation of all five zones. While for the cases of Ar-Ar and Ar-Ne all zones were visible, for the much heavier Xe the contact surface is almost nonexistent, which means that there were no longer discontinuities in the corresponding zones, and for the case of the much lighter He the shock and rarefaction waves have exchanged positions and the mixture is moving in the opposite direction. Although the above cases have already provided with a first insight in the mixing behavior, they were not adequate to provide specific pattern behavior so that safe conclusions to be drawn. For that reason two series of simulations were conducted. A gas species having all the properties of the monatomic noble gases was defined with mass $m=1 \times 10^{-26} \mathrm{Kg}$ and diameter $d=1 \times 10^{-10} \mathrm{~m}$ and considered as hard sphere. This gas will be referred to as the reference gas. For the first series of simulations the base configuration was investigated were the reference gas was positioned in both sides of the diaphragm and in each simulation the mass ratio was different while the diameter remained fixed. For the second series of simulations, following the same logic, the mass was fixed, while the diameter ratio varied. In that way, it was possible to investigate the mass and diameter influence unaffected from each other. 
Table 1. Cases simulated with noble gases

\begin{tabular}{ccc}
\hline Gas pairs & Mass ratio & Diameter ratio \\
\hline $\mathrm{Ar}-\mathrm{Ar}$ & 1.00 & 1.00 \\
$\mathrm{Ar}-\mathrm{Ne}$ & 1.98 & 1.33 \\
$\mathrm{Ar}-\mathrm{He}$ & 9.96 & 1.58 \\
$\mathrm{Ar}-\mathrm{Xe}$ & 0.30 & 0.64 \\
\hline
\end{tabular}

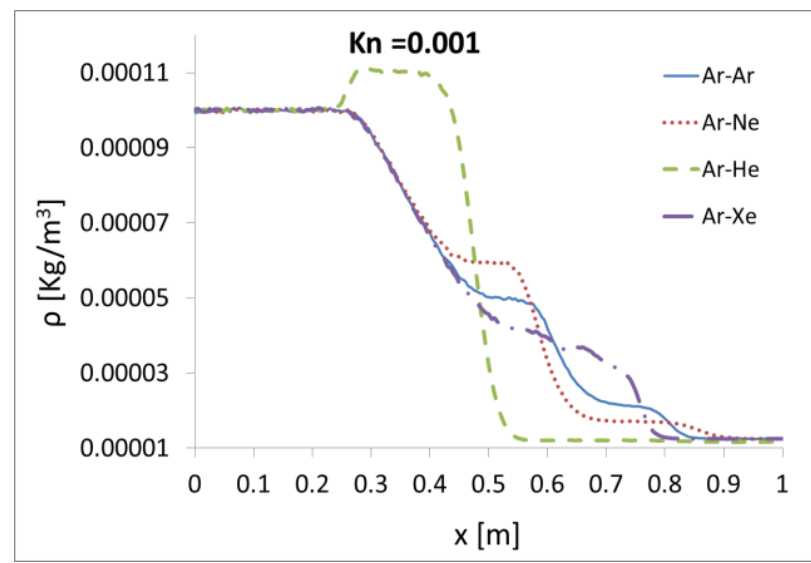

Figure 6. Mass density profiles for different pairs of noble gases at fixed Knudsen number equal to 0.001 as measured at the left side of the tube before the diaphragm is removed.

\section{Influence of the mass ratio}

The results below correspond to the base configuration with the reference gas having fixed diameter in all cases while the corresponding mass ratios between the species in the left and right side of the diaphragm are depicted in the legends of the Figures [7-11]. The solution corresponds to time $t=3 \times 10^{-4} \mathrm{sec}$. The exact mass ratios are the 1:1 corresponding to single gas case, 1:2, 1:3, 1:5 and 1:10 where the light gas is on the high density (left) side, and 2:1, 3:1, 5:1 and 10:1 where the light gas is on the low density (right) side. In Fig. 7 the mass density profiles provide the first set of results. For the cases with the solid lines when the mass on the right side is increased the standard downward step corresponding to the third zone, as observed in the classic Sod problem is gradually replaced with an upward step, this is firstly noticed in the case of $1: 5$ and it becomes clear in the case of 1:10. For the rest of the cases with opposite mass ratios it is interesting that the same behavior as in Fig. 6 with Ar-He is retrieved, firstly noticed at the case of 5:1 and became clearer at 10:1.

In Fig. 8 the molar fraction of the left species is depicted, when it is equal to one, the area is filled with pure left gas, while when equal to zero the area is filled with the right gas only. Focusing first on the solid lines it is seen that up until the position at $x=0.6 \mathrm{~m}$ the molar fraction is $C_{\text {Left }}=1$ which indicates that the mixing has not started yet, however, for the same position looking at Fig. 7 the mixture's density has been greatly reduced. This suggested that the mixing process did not start immediately after the diaphragm was removed neither did it happen at the middle position at $x=0.5 \mathrm{~m}$, in contrast, the light species initially diffused from the high to low pressure area without mixing rather pushing or rather compressing the heavier species further to the right. This behavior explained the increase in density that was observed for the cases 1:5 and 1:10 in Fig. 7 at the area $x \in(0.6,0.7)$. For the other set of cases with the dashed lines, the situation appears to be the opposite. The mixing process has started immediately and the greater the mass ratio the further had the light gas diffused from the low density area into the high density area with the heavy gas.

In Fig. 9 where the temperature profiles are presented, is worth noticing that for the mass ratios of 1:3, 1:5 and 1:10 the corresponding temperatures at the right end are higher than the fixed value in the initial undisturbed areas, the reason for this behavior is that for these cases and for the chosen solution time the heads of the shock waves had already reached the right boundary and had reflected backwards. The higher the temperature the further the shock had traveled in the opposite direction. Next, the velocity profiles in Fig. 10 shed more light into what happened with the cases of 5:1 and 10:1. It is seen especially for the latter ratio that there are regions that the mixture's velocity is negative explaining the high peek in density profiles approximately at $x \in(0.3,0.4)$. 


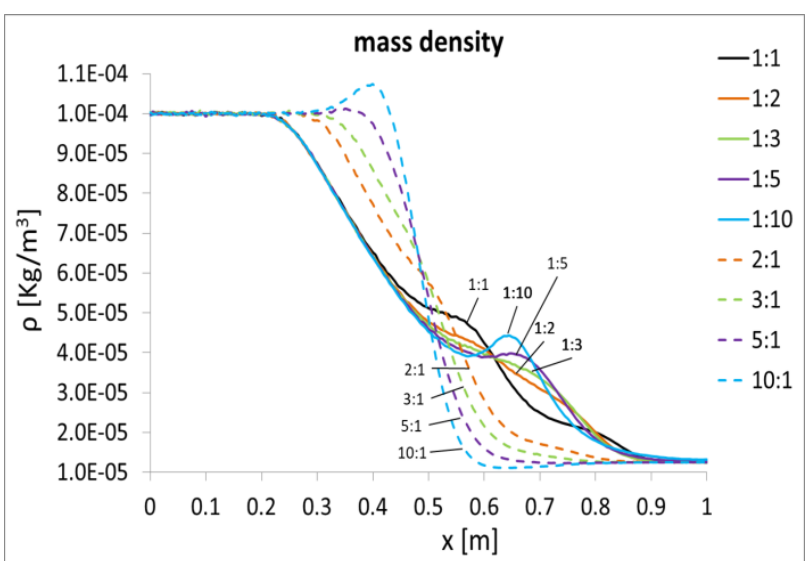

Figure 7. Mass density profiles for different mass ratios.

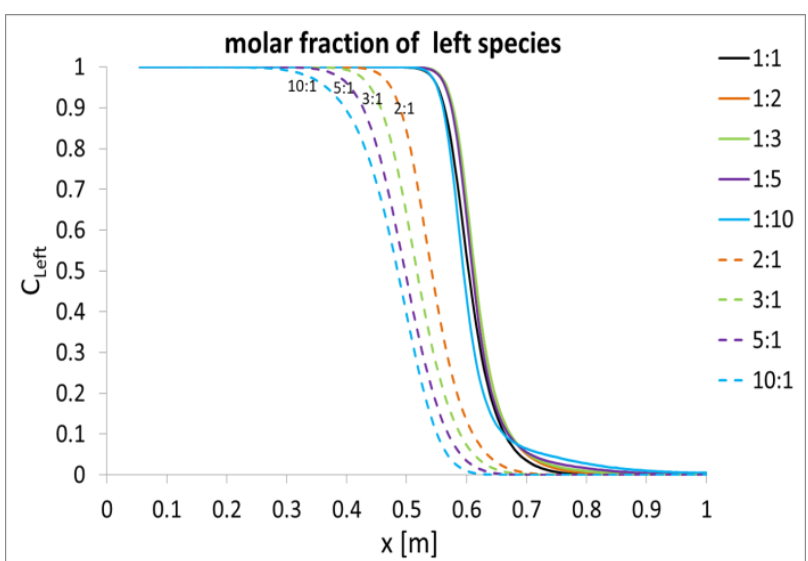

Figure 8. Molar fraction profiles for different mass ratios.

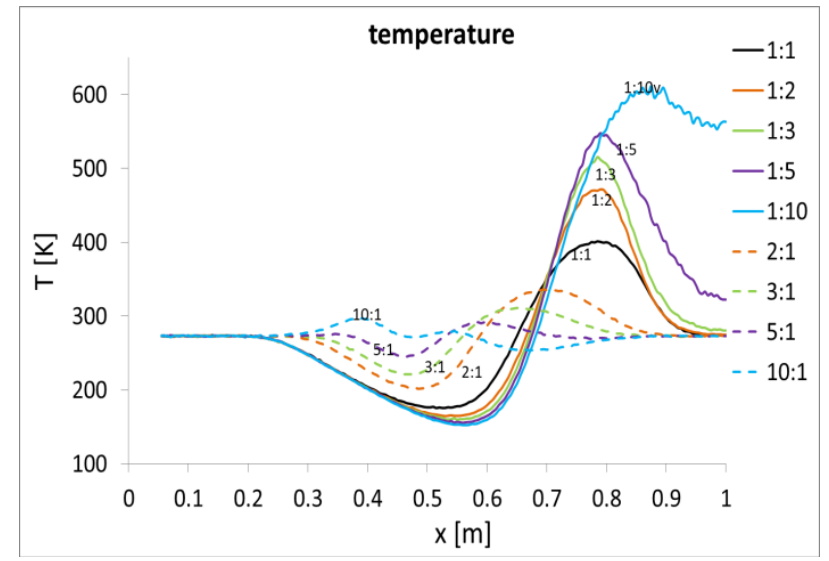

Figure 9. Temperature profiles for different mass ratios.

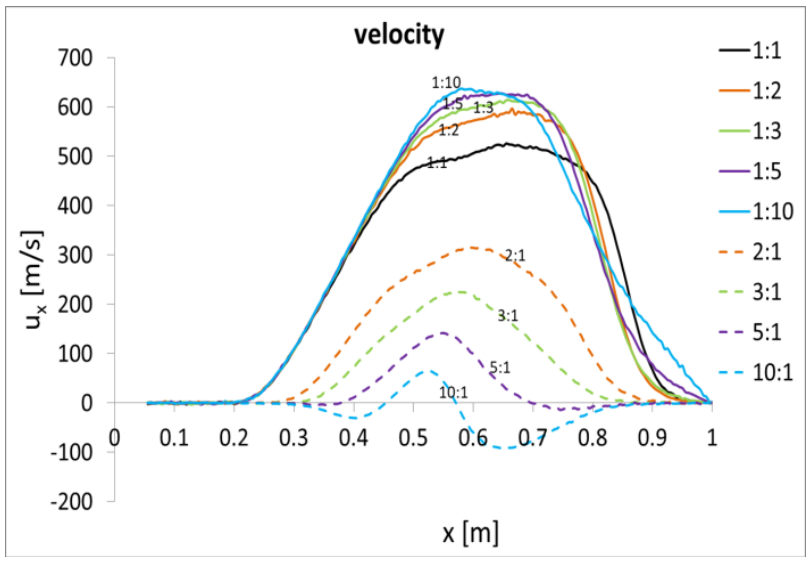

Figure 10. Velocity profiles for different mass ratios.

\section{Influence of diameter ratio}

Following the above logic as with the mass ratio results, the same base configuration is employed with the same reference gas, however, this time the species mass was fixed at both sides while their diameters were varying. In order to match the most common diameter differences met in real gases the ratios were chosen as 1:1 for the same species case, 1:1.25, 1:1.5, 1:1.75 and 1:2 when the smaller diameter species is on the (left) high density side and $1.25: 1,1.5: 1,1.75: 1,2: 1$ when the smaller diameter species is on the (right) low density side. A first observation on the figures suggested that diameter difference between species did influence the process of mixing, however in a much smaller scale than due to mass difference. In Fig. 12(a) and (b) the mass density plots are depicted for the cases when the smaller diameter species is on the high density side (a) and on the low density side (b) respectively. The main differences are spotted at regions 3 and 4 corresponding to the shock wave body. In specific, by focusing on the area approximately at $x \in(0.46,0.63)$, which corresponds to the area from the foot of the rarefaction to the contact discontinuity, the flow's transition was much smoother in (a) than in (b). However, at the other half of the shock wave, that is, the area approximately at $x \in(0.63,0.85)$, that is the area from the contact discontinuity until the head of the shock wave, the transition is much smoother in (b) than in (a). The above indicates that the wave 
formation and the distinction between the regions as seen in the analytical solution are replicated better with larger diameter particles than with smaller. In Fig. 13(a) and (b) the molar fraction of the left species is depicted for the smaller and larger diameter species respectively. The importance of these plots is twofold. First, they are almost identical, which indicates that the mixing process is affected in the same way regardless of the differences in diameter ratios between the two figures. Second, in both figures the molar fraction was reduced at the location of the diaphragm, which means that the mixing started immediately after the removal of the diaphragm, contrary to the case as shown before between the heavier and lighter species.

Finally, in Fig. 13(a) and (b) the velocity profiles are depicted for the two sets of diameter ratios respectively. Here, symmetry appears between the two figures covering the area between the regions 3 and 4 . That is regions 3 and 4 of Fig.13(a) are symmetrical to regions 4 and 3 of Fig.13(b) respectively. This behavior follows the same pattern as with the density profiles in Fig. 11.

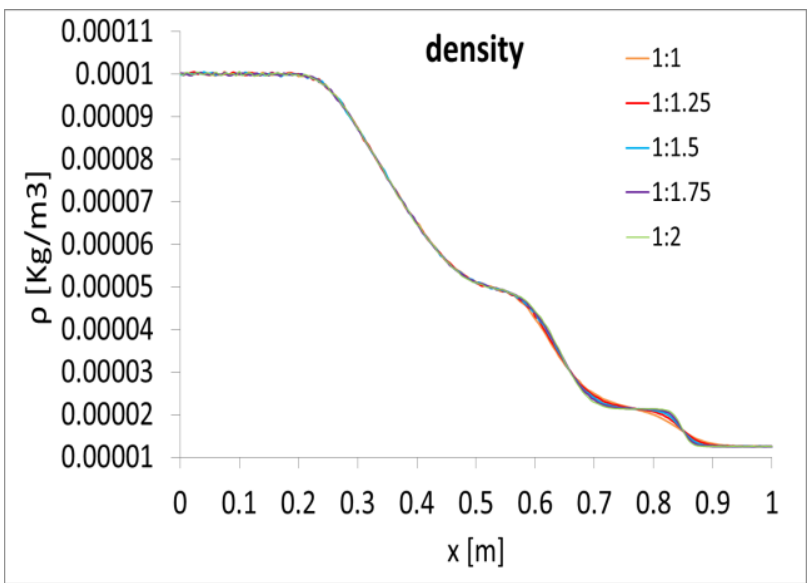

(a)

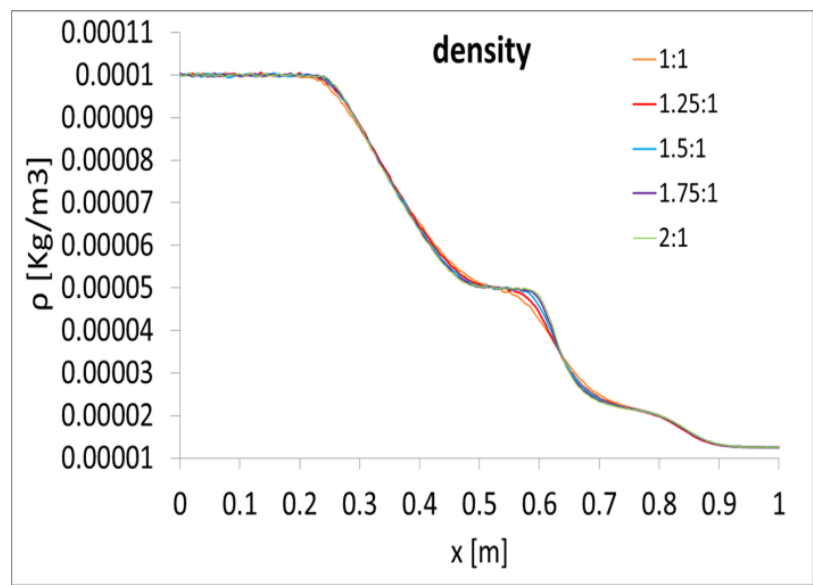

(b)

Figure 11. Mass density profiles for different diameter ratios when (a) the species with the smaller diameter is at the high density side and (b) when the species with the smaller diameter is at the lower density side.

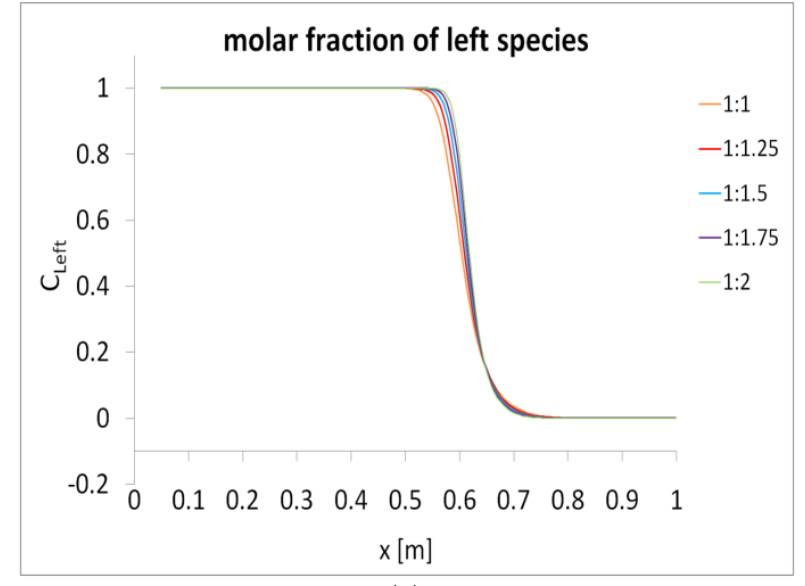

(a)

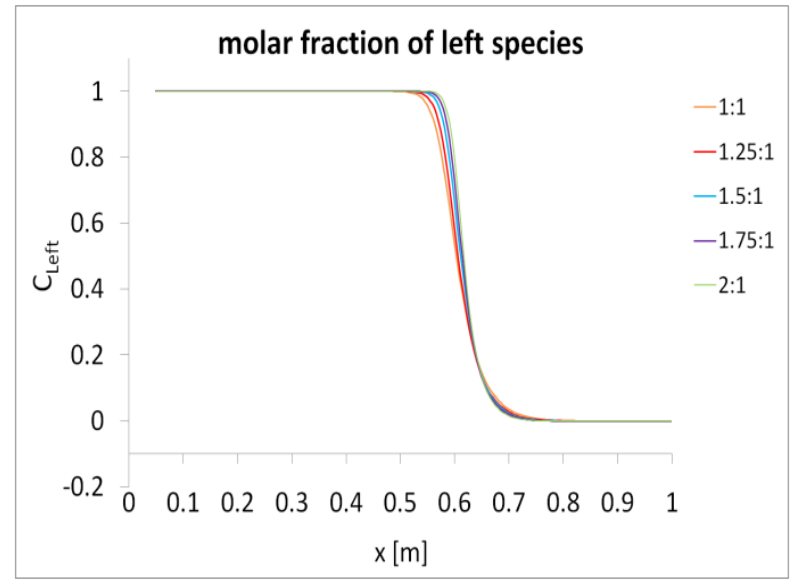

(b)

Figure 12. Molar fraction profiles of the left species when (a) the smaller diameter species is on the left side and (b) the larger diameter species is on the right side. 


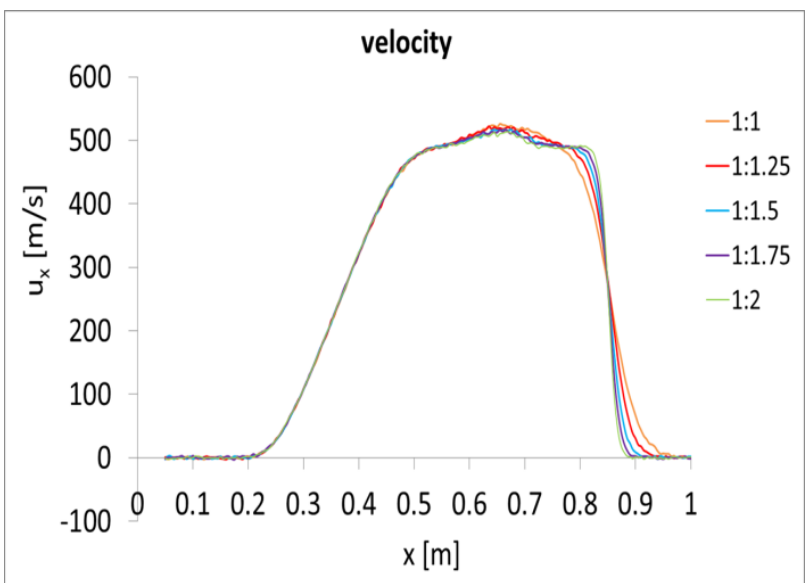

(a)

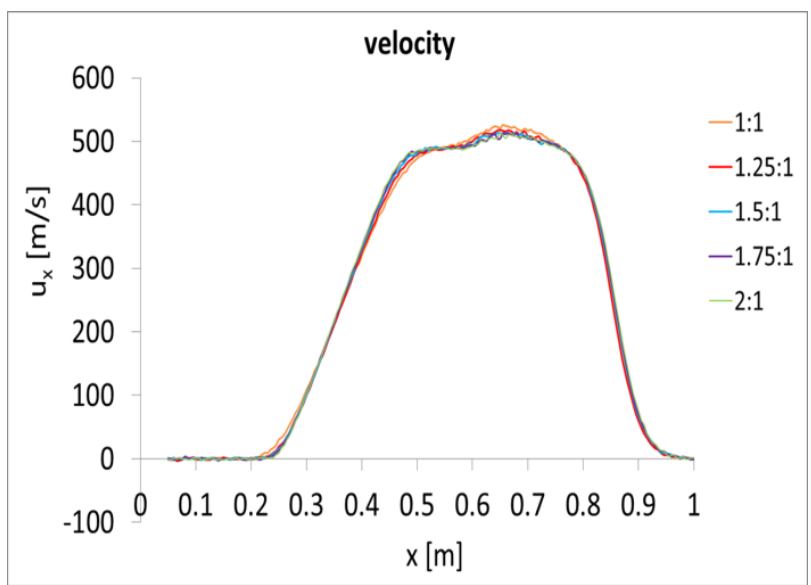

(b)

Figure 13. Velocity profiles for different diameter ratios when (a) the species with the smaller diameter is at the high density side and (b) when the species with the smaller diameter is at the lower density side.

\section{Influence of the molecular model}

In DSMC the most prevalent molecular models are the Hard Sphere (HS), the Variable Hard Sphere (VHS) and the Variable Soft Sphere (VSS). The HS model is the most simplistic one, physically it means that two particles cannot be closer than their diameter and when they are separated their interaction force is zero. However, it fails to provide the correct diameter of the species at different temperatures. The VHS model, introduce a hard sphere molecule but with a diameter that is a function of the translational molecular velocity, in that way it allows for the diameter to be corrected at each cell and time step [30]. A next step further from the VHS model is given by the VSS model in which the diameter varies in the same way as with the VHS model, however a scattering parameter is provided that corrects the deflection angle value to better match that of real gas values [31]. Due to its simplicity and low computational overhead the HS model is widely used especially in cases of single gas flows as well as of isothermal problems or at least in problems with very small temperature gradients.

In this section of results the application of the HS, VHS and VSS molecular models is presented for the cases of a single species and of a mixture in Fig. 14(a) and (b) respectively. The tests confirm that for the simple gas case in Fig.14(a) the results of all models coincide, while for the case of Ar-Ne in Fig 14(b) the VHS and VSS models give slightly different results in the most critical zones covering the area between the foot of the rarefaction wave until the head of the shock.

\section{CONCLUSION}

In this work the Sod-shock tube problem for gas mixtures was solved with the DSMC method. The goal was to investigate how the differences in masses and diameters between different species influence in general the mixing process and the formation of the waves. For that reason, two groups of tests were conducted, the first one that involved a series of simulations of gasses with different masses but fixed diameters and the second one another series of simulations that involved gasses with different diameters but fixed masses. Results showed that the mass difference was much more influential than diameter difference; in fact, the greater mass ratio is the higher is the effect on the flow formation. Finally, a comparison between the HS, VHS and VSS molecular models showed that for a single species case all models give the same results, while for mixtures the VHS and VSS gave slightly different results between the critical zones of the rarefaction and shock waves. 


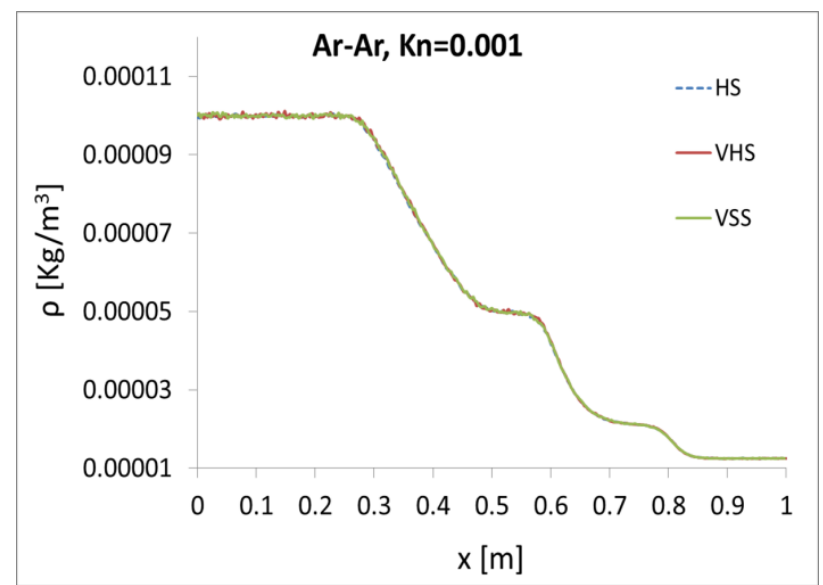

(a)

Figure 14. Comparison of mass density profiles corresponding to HS, VHS and VSS molecular models, for the single gas case of Argon.

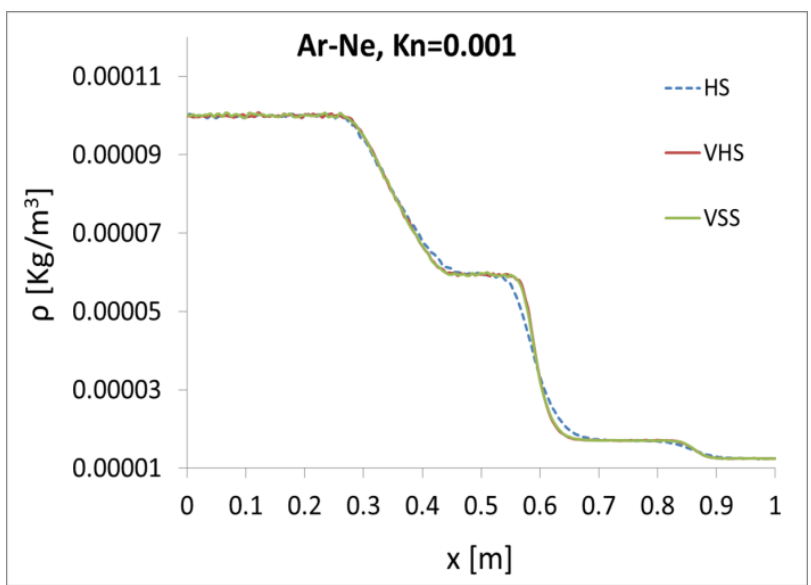

(b)

Figure 15. Comparison of mass density profiles corresponding to HS, VHS and VSS molecular models, for the case of Ar $\mathrm{Ne}$.

\section{ACKNOWLEDGMENTS}

This project has received funding from the European Union's Framework Programme for Research and Innovation Horizon 2020 (2014-2020) under the Marie Sklodowska-Curie Grant Agreement No. 643095. Also the second author S.S. would like to acknowledge the partial support provided by the Bulgarian NSF under grand DN $02 / 7-2016$.

\section{REFERENCES}

1. C.-Y. Lee, C.-L. Chang, Y.-N. Wang, and L.-M. Fu, IJMS 12, 3263 (2011).

2. A. Chaudhuri, A. Hadjadj, C. Guha, and T. K., in Recent Advances in Technologies 8, edited by M. A (InTech, 2009).

3. N.-T. Nguyen, "Mixing in Microscale," in Microfluidic Technologies for Miniaturized Analysis Systems (Springer US, Boston, MA, 2007), pp. 117-155.

4. G. López Quesada, G. Tatsios, D. Valougeorgis, M. Rojas-Cárdenas, L. Baldas, C. Barrot, and S. Colin, Micromachines 10, 249 (2019).

5. G. Tatsios, M.H. Vargas, S.K. Stefanov, and D. Valougeorgis, Heat Transfer Eng 37, 1085 (2016).

6. I.A. Graur, J.G. Méolans, and D.E. Zeitoun, Microfluid Nanofluid 2, 64 (2006).

7. M. Vargas, S. Stefanov, and V. Roussinov, International Journal of Heat and Mass Transfer 59, 302 (2013).

8. A. Tsimpoukis, C. Tantos, and D. Valougeorgis, J. Phys.: Conf. Ser. 785, 012008 (2017).

9. J. Chen, S.K. Stefanov, L. Baldas, and S. Colin, International Journal of Heat and Mass Transfer 99, 672 (2016).

10. S. Stefanov, P. Gospodinov, and C. Cercignani, Physics of Fluids 10, 289 (1998).

11. S.K. Stefanov, SIAM J. Sci. Comput. 33, 677 (2011).

12. J. Chen, S.K. Stefanov, L. Baldas, and S. Colin, International Journal of Heat and Mass Transfer 99, 672 (2016).

13. C. Tantos, D. Valougeorgis, International Journal of Heat and Mass Transfer 15 (2018).

14. M. Vargas, S. Stefanov, and V. Roussinov, International Journal of Heat and Mass Transfer 59, 302 (2013).

15. C. Cercignani and F. Sharipov, Physics of Fluids A: Fluid Dynamics 4, 1283 (1992).

16. S. Naris and D. Valougeorgis, D. Kalempa, and F. Sharipov, Phys. Fluids 17, 100607 (2005).

17. A.Ph. Polikarpov, M.T. Ho, and I. Graur, International Journal of Heat and Mass Transfer 101, 1292 (2016).

18. M. Wang and Z. Li, International Journal of Heat and Mass Transfer 49, 1696 (2006). 
19. M. Le and I. Hassan, Applied Thermal Engineering 27, 2370 (2007).

20. S. Meskos, S. Stefanov, and D. Valougeorgis, Micromachines 10, 178 (2019).

21. F. Sharipov, "Discrete Velocity Method" in Rarefied Gas Dynamics: Fundamentals for Research and Practice (Wiley-VCH, Weinhein, GE, 2015).

22. G.R. McNamara and G. Zanetti, Phys. Rev. Lett. 61, 2332 (1988).

23. G.A. Bird, Molecular Gas Dynamics and the Direct Simulation of Gas Flows (Clarendon press, Oxford, 1994)

24. G.A. Sod, Journal of Computational Physics 27, 1 (1978).

25. S. Tiwari, A. Klar, and S. Hardt, Journal of Computational Physics 228, 7109 (2009).

26. V.E. Ambrus and R. Blaga, Phys. Rev. C 98, 035201 (2018).

27. J.Y. Yang, J.C. Huang, and L. Tsuei, Proceedings of the Royal Society A: Mathematical, Physical and Engineering Sciences 448, 55 (1995).

28. Z.-H. Li and H.-X. Zhang, Journal of Computational Physics 193, 708 (2004).

29. S. Colin, J. Heat Transfer 134, 020908 (2012).

30. G.A. Bird, Prog. Astronaut. Aeronaut. 74, 239-255, 1981.

31. K. Koura and H. Matsumoto, Physics of Fluids A: Fluid Dynamics 3, 2459 (1991). 